\title{
A MATHEMATICAL FORMULATION OF URBAN ZONING SELECTION CRITERIA IN A DISTRIBUTED SERVICE NETWORK
}

\author{
Suchita Gupta ${ }^{1}$, Arun Pratap Singh ${ }^{2}$ \\ ${ }^{I}$ Department of Mathematics, Invertis University, Bareilly, UP, India \\ ${ }^{2}$ Department of Mathematics, Future Institute of Engg, \& Technology, Bareilly, UP, India
}

\begin{abstract}
Distributed Service Network is a wide term related to distribution and travelling i.e. distribution of resource among facilities located at various locations and travelling of resource along a distributed network. Policy making problems in distributed service networks can be clearly classified into a number of hierarchical levels. The levels are distinguished by time horizon of the problem, by amount of cost involved in the implementation of a solution, and by the political implications of the solution. In the public sector, it includes ambulance, fire, police and other services. In the private sector courier, taxi, repair, maintenance and the like are considered to be distributed service network.
\end{abstract}

In this paper an attempt has been made to obtain a mathematical model by which a network can be partitioned into subnetworks in a Distributed Service Network. The constraints like demand equity, contiguity, compactness, enclaves etc. are applied for Zoning.

Keywords: Zoning, Contiguity, subnetwork, Compactness, Enclave. - ***

\section{INTRODUCTION}

Let us suppose that there are nine nodes in a network and we want to divide the network into four zones. We know the demand of each node and the distance between the nodes in units of time. We impose the constraints that the ideal demand generated in each zones should be $25 \%$, however, we allow a zone should not exceed 10 units of time. We make zones by including nodes under the condition of constraints of demand and the largest shortest distance.

\section{ZONING}

The process by which a network is partitioned into smaller networks each of which is delegated with a certain degree of autonomy in terms of resource allocation and operation is known as Zoning. Reasonable criteria of Zoning are response time minimization, equity, compactness.

A network $\mathrm{G}(\mathrm{N}, \mathrm{L})$ where $\mathrm{N}$ is the set of demand nodes and $\mathrm{L}$ is the set of links, must be partitioned into $\mathrm{M}$ Zones (districts ) $N_{j}, \mathrm{j}=1, \ldots, \mathrm{M}$. The zones must be mutually exclusive and collectively exhaustive in the sense that:

$M$

$\cap N_{j}=\varphi$

$\mathrm{j}=1$

$\bigcup_{j=1}^{M} N_{j}=N$

Zoning Criteria: There are many steps involve in zoning criteria:

Demand Equity: In the context of providing services, particularly in the public sector, the concept of equity asserts that the entire population of potential clients be treated as equally as possible in term of quality of service they get.

Given that the fraction of demand is $h_{i}, i \in N\left(\sum_{i=N} h_{i}=1\right)$ equity as asserts if for every Zone $N_{j}$,

$$
\left|\sum_{i=N} h_{i}-\frac{1}{M}\right| \leq \alpha / M
$$

where $\alpha, 0<\alpha<1$, is the maximum allowable deviation of the demand of the zone from the average demand and $\mathrm{M}$ be the desired number of subnetworks.

The ideal demand generated in each zone should be $25 \%$. However, we allow for $2.5 \%$ deviation; namely, an acceptable zone may generate demand ranging from $22.5 \%$ to $27.5 \%$ of the total demand.

Contiguity: The basic principle in Zoning is contiguity. A subnetwork is contiguous if it is possible to travel from every node in subnetwork to every other node in it without crossing another subnetwork. The possible way to illustrate contiguity is by constructing a square matrix whose elements are binary. Considering a square matrix $\mathrm{B}$ of $\operatorname{order}(n \times n)$, where:

$$
B_{i k}=\left[\begin{array}{cr}
1 & \text { if nodes } i \text { and } k \text { connected by a link } \\
0 & \text { otherwise }
\end{array}\right.
$$

Compactness: An intuitive interpretation of the notion compactness that edges of a zone are not too remote each other. In partitioning a planar area (rather than a network) compactness can be measured by ant three measurements: 
- Resemblance of the zone to a square

- Resemblance of the zone to a circle

- "Reasonable" distance of the population from the centre of the zone.

A zone $N_{j}$ is compact if the shortest distance between two nodes $\mathrm{a}$ and $\mathrm{b}$ is $N_{j}$ is less than or equal to $\beta-\mathrm{a}$ predetermined constant called "exclusion distance". Let $\mathrm{E}$ be a square matrix of order $(n \times n)$ when:

$$
E_{j k}=\left[\begin{array}{rr}
1 & \text { if } d(i, k) \leq \beta \\
0 & \text { otherwise }
\end{array}\right.
$$

Where $\mathrm{d}(\mathrm{i}, \mathrm{k})$ is the shortest distance between $\mathrm{i}$ and $\mathrm{k}$, zone $N_{j}$ is compact if for any a,b $\in N_{j}$

$$
E_{a b}=[d(a, b) \leq \beta]
$$

Enclaves: An enclave is a node, or subsets of nodes that can not constitute an independent zone because of equity criteria, on the other hand, the nodes cannot be connected to other "free"( not - yet - selected)nodes for non- contiguity reasons. During the process of Zoning we have to make sure that we do not create enclaves.

Natural Boundaries: Natural and geographical boundaries can certainly impose constraints on a zoning process. Other boundaries may include administrative boundaries. However, we claim that such boundaries are inherent to the network topology, because the link lengths do not read as aerial distances but they do reflect the realistic access time (or distance) to a node. Therefore, we are not required to incorporate such extra measure into the Zoning algorithm.

\section{MATHEMATICAL FORMULATION:}

Suppose there are $\mathrm{S}$ feasible zones (in terms of the requirements on equity, contiguity, compactness and others). Let $X_{j}$ be a binary variable

$$
\boldsymbol{X}_{\boldsymbol{j}}=\left[\begin{array}{rr}
1 & \text { if zone } \mathrm{j} \text { is selected } \\
0 & \text { otherwise }
\end{array}\right.
$$

The objective function of zoning is defined as:

Min $\quad \operatorname{Max} C_{j} X_{j}$

$\mathrm{j}=1, \ldots ., \mathrm{S}$

Subject to

$$
\sum_{j=1}^{S} a_{i j} X_{j}=1 ; i=1, \ldots \ldots, n
$$

$$
\begin{gathered}
\sum_{j=1}^{s} X_{j}=M \\
\mathrm{X}_{\mathrm{j}}=0,1 ; j=1, \ldots \ldots, . S
\end{gathered}
$$

and $\mathrm{C}=\left|\sum_{i \in N_{j}} h_{i}-\frac{1}{M}\right| \leq \alpha / M$ is the relative deviation of zone $N_{j}$ 's demand from $1 / \mathrm{M}$.

In (1), we minimize the maximum relative deviation subject to the constraints that ensure (1) that each node is assigned to only one zone (2), (2) that $\mathrm{M}$ zones are eventually being selected (3), and (3) that each $X_{j}$ can have only the value 0,1 (4).

The objective function can also be defined as:

$$
\operatorname{Min} \sum_{j=1}^{S} d_{j} X_{j}
$$

Where $d_{j}$ is the maximum shortest distance between any two nodes in $N_{j}$.

Where

$$
\boldsymbol{a}_{i j}=\left[\begin{array}{cr}
1 & \text { if node } \mathrm{i} \text { is in zone } \mathrm{N}_{\mathrm{j}} \\
0 & \text { otherwise }
\end{array}\right.
$$




\section{A PICTORIAL REPRESENTATION:}

Now we want to divide the network $G$ into four subnetworks:



Let us start with node 1 , it is linked to node 2 ; together they accumulate $27 \%$ of the total demand. They do not violate compactness, nor do they enclave any node; thus $\{1,2\}$ constitutes a feasible zone. We cannot add any more node to $\{1,2\}$, since any additional node that is linked either to 1 or to 2 will push the demand beyond the tolerated limit, which is $27.5 \%$. Node $\{1,3\}$ from a feasible zone by similar argument and cannot be further argumented.

Now, we turn to node 2 . New feasible zones are $\{2,4\}$ and $\{2,5,9\}$. When node 3 is examined, the only new feasible zone is $\{3$, $6\}$. The zone $\{3,7\}$ could be feasible in terms of equity $(23.5 \%)$, contiguity and compactness. However if the zone is established node 6 becomes an enclave. Hence, we exclude $\{3,7\}$ from the set of feasible zones.

\begin{tabular}{|c|c|c|c|c|c|}
\hline Zone No. & Nodes & Demand \% & Relative Deviation & $\begin{array}{ll}\begin{array}{l}\text { Largest } \\
\text { Distance }\end{array} & \text { shortest } \\
\end{array}$ & Selection (List) \\
\hline 01 & 1,2 & 27 & 0.8 & 5 & \\
\hline 02 & 1,3 & 24 & 0.4 & 3 & 1 \\
\hline 03 & 2,4 & 24.5 & 0.2 & 4 & 2 \\
\hline 04 & $2,5,9$ & 26 & 0.4 & 8 & \\
\hline 05 & 3,6 & 22.5 & 1.0 & 6 & 3 \\
\hline 06 & $4,5,9$ & 24.5 & 0.2 & 8 & 4 \\
\hline 07 & 4,7 & 25 & 0 & 9 & \\
\hline 08 & 4,8 & 24 & 0.4 & 8 & \\
\hline 09 & $5,8,9$ & 25.5 & 0.2 & 8 & 5 \\
\hline 10 & 6,7 & 26 & 0.4 & 3 & 6 \\
\hline 11 & 7,8 & 26 & 0.4 & 6 & 7 \\
\hline
\end{tabular}


Now, we suppose we minimize the total relative deviation. We start at the first section of given table, namely all the zones whose root node is node 1 . We call this set of zones list 1 . Among them, zone 2 is preferred since it incurs a relative deviation 0.4 . So, we mark zone 2, tentatively, as a candidate zone and we record that node 1 and 3 are now covered.

Since node 2 is not covered, we have to select a zone from the second section list 2, while avoiding double coverage. A preferred candidate zone is 3 , whose deviation is equal to 0.2 . The list of covered nodes now includes $\{1,2,3,4\}$.

The next not-covered node is 5 , so we turn to the fifth section list 5 of the table and zone 9 to our candidate set. The covered nodes are $\{1,2,3,4,5,8,9\}$.

We now have to examine node 6 and select zone 10 to the candidate partitioning. All the nodes are now covered and the partitioning is mutually exclusive. The candidate zones are $\{2,3,9,10\}$, the total deviation is 1.2 , hence average deviation is $1.2 / 4$ $=0.3$.

The zones $\{2,3,9,10\}$ constitute the optimal partitioning.

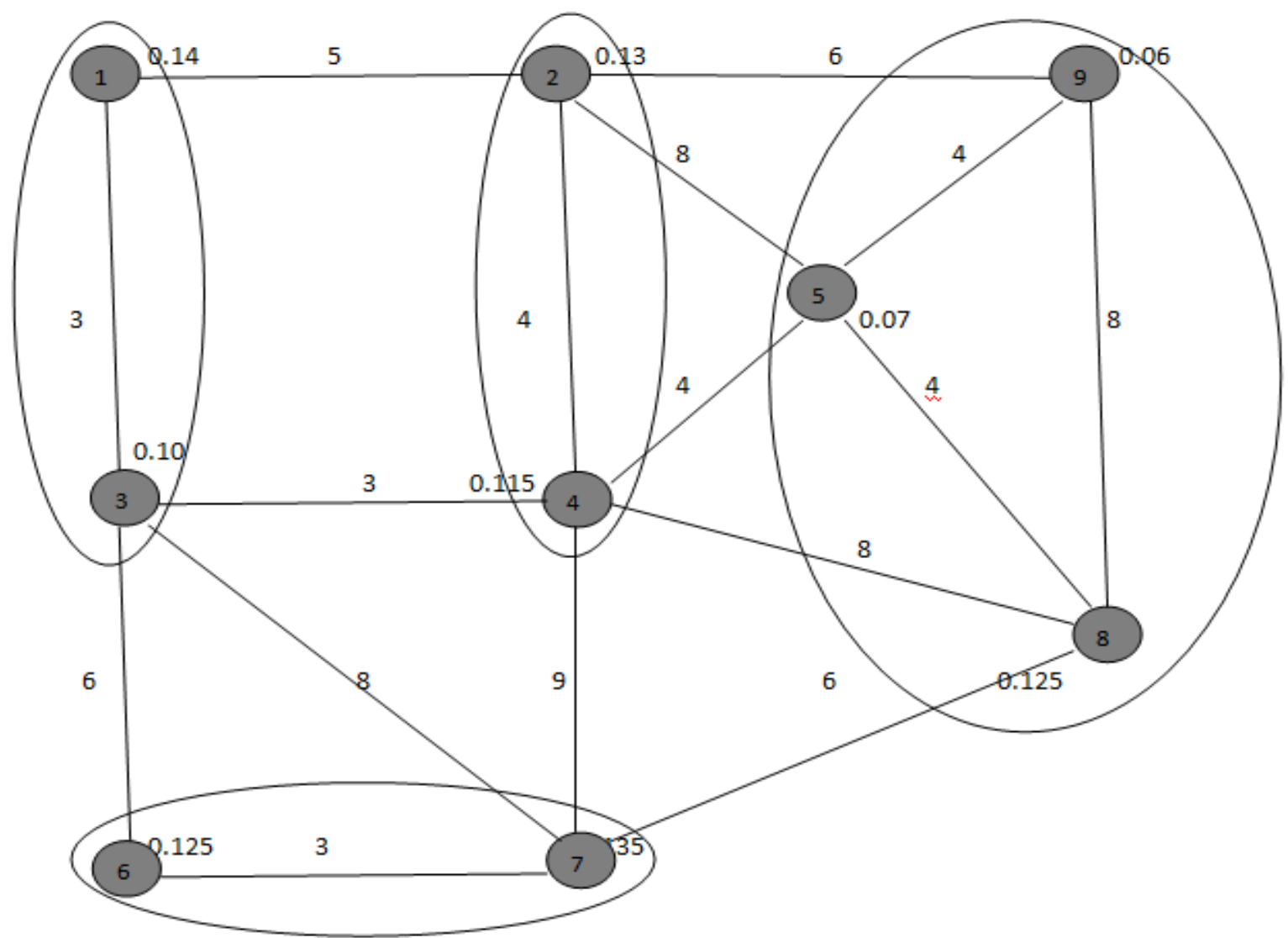

\section{CONCLUSION}

In the public sector like ambulance, fire, police and the other services and in the private sector like courier, taxi, repair, maintenance etc., when we start a service policy, our first aim is to partition a network, into smaller subnetworks. By partitioning a network into subnetworks, we can provide service to the public easily. Here the term "Net-work" connotes geographical distributions namely different places located in a certain area, these are called Nodes. Nodes can be cities in a state, blocks within a neighbourhood, a police headquarter or police check post for a specified area etc. With the help of the mathematical model, we can partition a network into subnetworks.

\section{REFERENCES}

[1] Richard C. Larson, Amedeo R. Odoni, "Urban Operation Research", 1981.

[2] Ahituv, N, and O. Berman "Quantitative Models for Policy Making in Urban Emergency Networks", International Journal of Policy and Information, 6(2), pp. 35-42 (1982).

[3] Battey, M., (1971),"Modelling cities and Dynamic Systems", Nature, 321, pp 425-428 (1976),'Urban Modelling, Algorithms, Calibrations, Predictions", Cambridge University Press, Cambridge, England (1979). 
[4] Battey, M., Bourke, R. Cormode, Anderson, P and M, Nicholis,"Experiments in Urban Modelling for Country Structure Planning : The Area of 8 Pilot Model", Environment and Planning A, 6, pp. 455478 (1974).

[5] Garfinkel, R.S. and G.L. Nemhauser,"Optimal Districting by Implicit Enumeration Teachnique", Mgmi. Sci. 16, pp. 495-508 (1970).

[6] Mirchandani, P.B.,"Analysis of Stochastic Networks in emergency Service System", IRP. TR-15-75. Operations Research Centre, Massachusetts Institute of Teachnology, Cambridge, Mass.(1975).

[7] Battey, M, and I. Masser,'Spatial Decompositions and partitions in Urban Modelling", in Cripps, E.L.,(ed.), Regional Science-New Concepts and Old problems, London Papers in Regional Science, 5, Pion, London pp. 188-206 (1975).

[8] Singh, A.P, "Innovative Resource Planning Project in Distributed Service Network, An Operational Research Apporach", (2000). 